\title{
Cochlear Implants in Single-Sided Deafness
}

\author{
Christopher K. Giardina • Eric J. Formeister • \\ Oliver F. Adunka
}

Published online: 18 October 2014

(C) Springer Science + Business Media New York 2014

\begin{abstract}
Single-sided deafness presents a unique challenge to otolaryngologists and audiologists. While the normal hearing ear may allow listeners to perform adequately on audiometric screening, individuals with only one functioning cochlea suffer when resolving speech in noisy environments and in sound localization - which both contribute to a reduced quality of life. Though there are a variety of strategies that provide contralateral routing of sound signals, the cochlear implant is the only treatment to truly restore binaural hearing. Only very recently has cochlear implantation (CI) for singlesided deafness begun in earnest, with encouraging results that demonstrate the strengths and pitfalls of implantation over traditional extracochlear methods. The purpose of this review is to update the field by emphasizing binaural benefits, discussing historical treatments of single-sided deafness, critically evaluating recent data on outcomes of CI for single-sided deafness, and recommending indications for cochlear implants in single-sided deafness in children, adults, and subjects with concurrent ipsilateral tinnitus.
\end{abstract}

Keywords Single-sided deafness - Unilateral cochlear implant - Speech in noise - Sound localization .

Binaural hearing $\cdot$ Unilateral tinnitus

This article is part of the Topical Collection on Ear Surgery.

C. K. Giardina · E. J. Formeister

Department of Otolaryngology/Head and Neck Surgery,

University of North Carolina School of Medicine, Chapel Hill,

NC, USA

O. F. Adunka $(\square)$

Department of Otolaryngology/Head and Neck Surgery,

The Ohio State University Wexner Medical Center, Columbus, OH 43210, USA

e-mail: oliver.adunka@osumc.edu

\section{Introduction}

Single-Sided Deafness

Single-sided sensorineural deafness, hereafter referred to as SSD, is defined as a severe-to-profound hearing loss ( $>70 \mathrm{~dB}$ normal hearing level, nHL) in one ear with preserved audiometric thresholds in the contralateral ear. Though the precise incidence and prevalence of SSD is unknown, its prevalence has been estimated anywhere from 3 to $6 \%$ of the population [1], and among children and teenagers ranges from 2 to $5 / 1000[2 \bullet \bullet]$. Incidence is estimated at 1 in 3,700 newborns being born with congenital SSD [3] and the incidence increases with age [4]. Sudden sensorineural hearing loss (SSNHL) is the most common cause of SSD, though a myriad of other causes are known, including intractable Ménière's disease, unilateral vestibular schwannoma, trauma leading to temporal bone fractures, unilateral noise damage, and ototoxic drug exposure.

Historically, hearing limitations posed by SSD were largely understated, as it was assumed that one normal hearing ear provided sufficient auditory input for a relatively normal hearing experience [5]. It is now widely known that individuals with SSD face significant challenges to hearing in complex or noisy environments and in localizing or lateralizing sound sources [6॰]. Not surprisingly, individuals who suffer from SSD consistently rate lower in quality of life inventories such as the speech, spatial, and qualities hearing scale (SSQ) [7-10]. Deficiencies in speech comprehension are chiefly a result of impaired binaural squelch and summation, whereas deficiencies in azimuth localization are mostly due to impaired calculation of interaural phase differences (IPDs), time differences, and intensity differences $[6 \bullet, 7,10,11 \bullet \bullet]$. 


\section{Binaural Benefit to Speech Perception}

Speech comprehension is vastly benefited from having two separate sound inputs, specifically with respect to binaural squelch and summation. The squelch effect describes the ability of the brain to selectively filter noise from a desired sound when there is binaural hearing input, particularly in a hearing environment where noise and speech are originating from different azimuthal or vertical locations, due to interaural level, phase, and time differences. The advantage conferred with binaural hearing due to squelch is a $2-5 \mathrm{~dB}$ gain in signal-to-noise ratio (SNR) in speech discrimination testing [6•, 7]. Binaural summation is a psychoacoustic phenomenon wherein one acoustic stimulus reaching two ears provides the brain with redundant information that adds to the net perceived sound. This provides an additive effect to higher-order processing centers and results in a 2-6 dB improvement in speech reception thresholds compared to the monaural condition $[6 \bullet, 7]$. The head shadow effect refers to the reduction in intensity of sound reaching an ear opposite to the side of the sound source because the head functions as a physical attenuator. In subjects with SSD, speech and other sounds projecting directly at the deaf ear are primarily detected at the functioning contralateral cochlea. However, because of the head shadow effect, these sounds are up to $10-16 \mathrm{~dB}$ lower than the signal source, which makes speech more difficult to understand, especially with ambient background noise [7]. In addition to diminished sound levels at the contralateral hearing ear, limitations in comparing interaural sound intensity levels also affect a subject's ability to localize sounds.

\section{Binaural Input Necessary for Horizontal Sound Localization}

Sounds produced at an azimuth off-axis will arrive in each ear at slightly different times, at slightly different phases, and due to the head shadow effect, at slightly different intensities. Different binaural mechanisms are used to help localize sounds in the horizontal plane, depending on the acoustic signal quality (Table 1). For low stimulus frequencies, auditory phase resolution is robust enough to allow cross-correlation of interaural phase differences in order to infer the azimuthal location [6•]. The ability to differentiate between phases is maximized when the peakto-peak distance of a single wave spans the largest physical range of place-coded neurons in the superior olivary complex. As such, IPD is best suited to localize low-frequency sources and becomes less effective at frequencies greater than $800 \mathrm{~Hz}$, at an approximate wavelength that is shorter than twice the interaural distance of the human head [6•]. For acoustic stimuli greater than $16 \mathrm{kHz}$, phase resolution is nearly impossible. As such, the time delay between signal
Table 1 Several independent mechanisms of binaural integration assist in horizontal sound localization, depending on the acoustic stimulus.

\begin{tabular}{ll}
\hline Acoustic quality & Binaural azimuth localization mechanism \\
\hline $\begin{array}{l}\text { Low frequency } \\
(<800 \mathrm{~Hz})\end{array}$ & Interaural phase difference \\
$\begin{array}{c}\text { Mid frequency }(800 \mathrm{~Hz} \\
\text { to } 16 \mathrm{kHz})\end{array}$ & $\begin{array}{c}\text { Combination of interaural phase difference } \\
\text { and interaural time difference }\end{array}$ \\
High frequency & Interaural time difference \\
$(>16 \mathrm{kHz})$ & \\
High intensity (any F) & Interaural level difference \\
\hline
\end{tabular}

Summarized from content in $\left[6^{\bullet}\right]$

detection of each ear, called the interaural time difference (ITD), helps inter the sound source in the horizontal plane. Frequencies within the range of $800 \mathrm{~Hz}$ to $16 \mathrm{kHz}$ use a combination of IPD and ITD. In addition to IPD and ITD, larger differences in sound intensity between ears correlate with larger azimuths in a frequency-dependent manner. A normally functioning auditory system will expect attenuation between ears because of the head shadow attenuation and correlate larger interaural intensity differences with larger azimuth angles. Without two functioning cochleae, the head shadow effect, along with other binaural mechanisms, are ineffective in aiding horizontal localization.

\section{Statement of Purpose}

In the context of a recently changing political and healthcare climate, cochlear implantation (CI) candidacy has expanded to include subjects with relatively preserved hearing thresholds but reduced speech perception (Hybrid CI or electric acoustic stimulation). Demonstrable benefits of hearing in noise, sound localization, and quality of life from studies on CI for SSD similarly argue for an expansion of the indications for implantation. The purpose of this review is to critically evaluate the most recent literature on CI for SSD and to form conclusions regarding CI for subjects with SSD that will inform clinical practice and policy makers. Though the focus of this report is not to investigate the efficacy of unilateral CI for tinnitus reduction, because many of the studies were conducted with subjects who suffered from SSD and concomitant ipsilateral tinnitus, some discussion of the efficacy of CI for tinnitus reduction will follow as well.

\section{Treatments for Single-Sided Deafness}

\section{Historical Treatments}

In the past, treatment modalities to restore binaural hearing relied on amplification devices that transmit sound, through 
air or bone, from the deafened ear to the contralateral, normal ear. Such technologies include air conduction contralateral routing of sound (CROS), transcranial CROS ( $\mathrm{t}-\mathrm{CROS}$ ), and bone conduction technologies including the Bone-Anchored Hearing Appliance (Baha, Cochlear, Inc, Macquarie University, NSW, Australia, both percutaneous and transcutaneous solutions), the Sophono device (Sophono, Inc, Boulder, CO, USA), and the SoundBite dental conduction device (Sonitus Medical, Inc, San Mateo, CA, USA). An air conduction CROS hearing aid (HA) is decades-old technology that consists of a microphone placed in the deafened ear which transmits sound via a wire or wirelessly to a receiver that is placed in the normal hearing aid, thereby averting the negative head shadow effect in monaural listeners. Due to inexpensiveness, and ease of use and fitting, CROS hearing aids typically are the first line intervention for SSD [12]. Though users have endorsed subjective benefits from restoring the head shadow effect by allowing sound awareness from the deaf side $[6 \bullet, 12$, 13], this success has been tempered by its drawbacks, including the need to occlude the better ear canal and relatively poor overall improvement in hearing experience, particularly in regard to hearing in noise and sound localization [13, 14]. Transcranial CROS transmits a signal received by an air conduction hearing aid in the deaf ear to the contralateral cochlea via bone conduction. Theoretically, transcranial CROS represents an improvement over air conduction CROS because of a smaller reduction in the sound signal across bone versus electronically or wirelessly $[6 \bullet, 12]$. However, its use is not well established because there are only a limited number of trials with small sample sizes and conflicting data regarding improvements in speech perception, sound localization, and patient satisfaction relative to air conduction CROS [15, 16].

More recently, the Baha has gained traction for auditory rehabilitation in SSD. The Baha is a type of osseointegrated bone conduction device wherein an osseointegrated titanium implant is placed into the skull. An external sound processer transmits signals from the deafened ear through the titanium implant and eventually to the normal hearing cochlea on the contralateral side via bone conduction. In contrast to t-CROS, there is a voluminous literature on the efficacy of Baha. In addition to consistent reports of patient satisfaction and increased speech perception in noise, studies show moderate improvements in sound localization ability after Baha [17-19]. In comparative studies on the efficacy of Baha versus CROS, investigators have uniformly recommended Baha over CROS or t-CROS for the management of SSD [20-22]. While hearing aid and conduction technologies simply transfer sounds to the single functioning cochlea, CI in the deaf ear uniquely affords the opportunity to utilize two independent cochlear generators.
Cochlear Implantation in Single-Sided Deafness

Despite the improvement in hearing in noise and localization reported by Baha and CROS users, CI implantation holds promise as the definitive treatment for SSD. Although the first studies of unilateral CI implantation, dating back to 1957 , were in patients with bilateral deafness [23-25], CI for SSD began fortuitously in the 2000s as an experimental treatment for incapacitating and intractable tinnitus [26] when users reported marked gains in sound localization and speech perception in complex listening environments [7]. Because hearing loss co-exists in up to $85 \%$ of individuals with tinnitus [27], hearing improvements were likewise reported in subsequent studies of CI in tinnitus patients with ipsilateral SSD [9, 10, 28]. The small number of studies that directly compare benefits with CI versus Baha or CROS strongly endorse CI over these more rudimentary treatment strategies [10, 29]. A number of studies have since reported on substantial improvements in sound localization, hearing in noise, and quality of life measures due to restoration of binaural squelch, summation, and the head shadow effect $[6 \bullet, 8,10$, 30]. Despite these advances, candidate selection and anticipated benefits are not as clear and predictable as implanting a bilaterally deaf individual.

\section{Speech Benefits after Unilateral Cochlear Implantation}

Several recent studies investigate the utility of CI for SSD in both pediatric (Table 2) and adult (Table 3) populations. In short, the main benefits for speech perception with regard to a unilateral implant for SSD are an increased speech understanding, especially in noisy environments, and a decreased effort to hear [6•, 11••].

When solely analyzing the SSD ear, Hansen et al. report a $28 \%$ increase in CNC scores and a $40 \%$ increase in AzBio sentences when comparing unilateral free-field audio stimulation pre-implantation to electrical CI stimulation post-implantation [31]. Despite this increase in cochlear function of the SSD ear, the binaural benefits of free-field speech comprehension gleaned after implantation are limited in quiet environments, likely because the normal hearing ear doesn't need to rely on binaural squelch and summation when noise is not present. In a case series with 3 subjects implanted for SSD, Firszt et al. reported only 1 patient who showed statistically significant improvements in CNC speech scores in the bimodal, quiet condition compared to either unilateral $\mathrm{NH}$ or $\mathrm{CI}$-alone conditions [8]. In the same study, adding noise to the speech tests resulted in $2 / 3$ patients showing significantly better CNC scores in the bilateral condition than solely with either the normal hearing ear alone. 


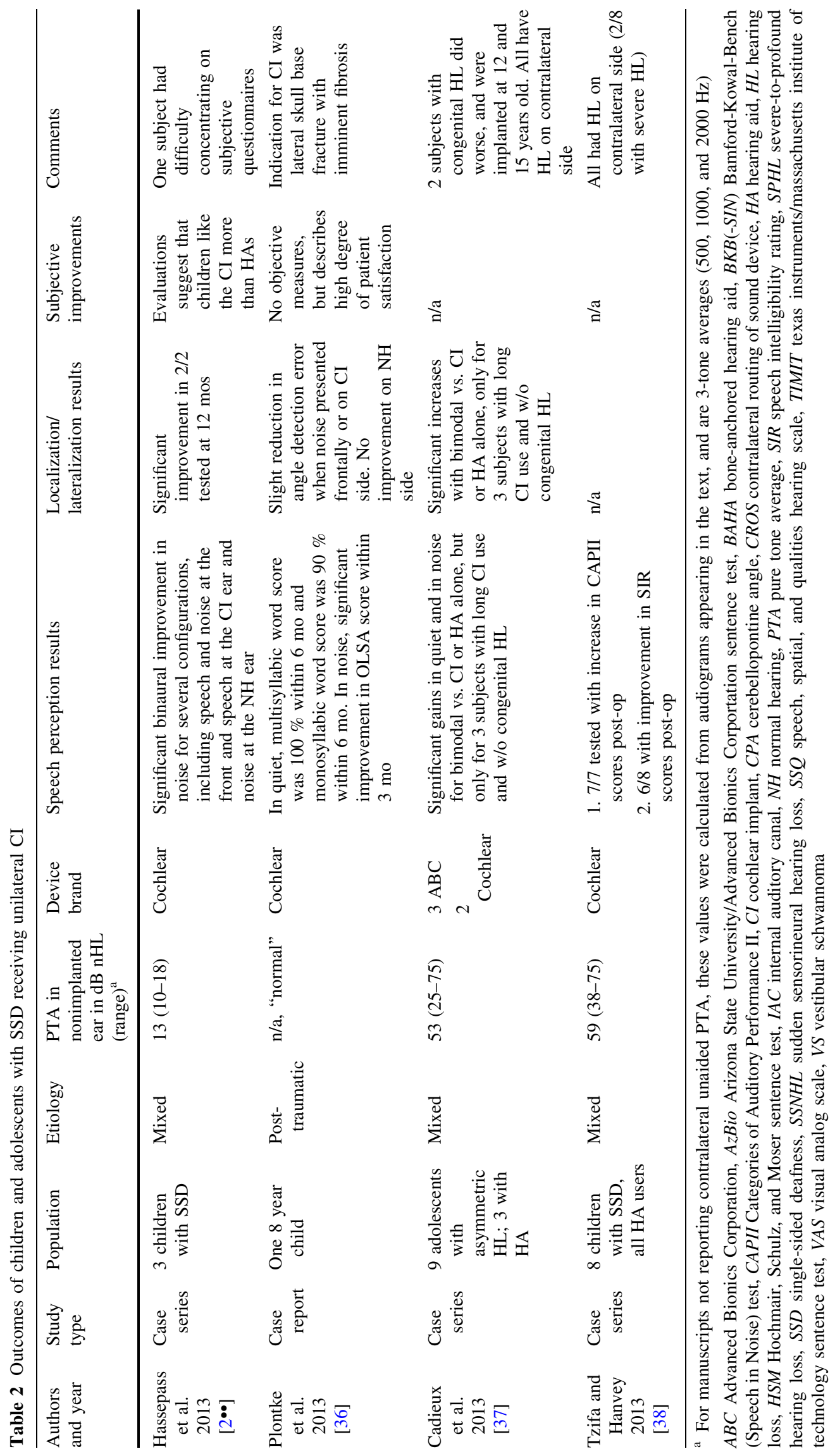




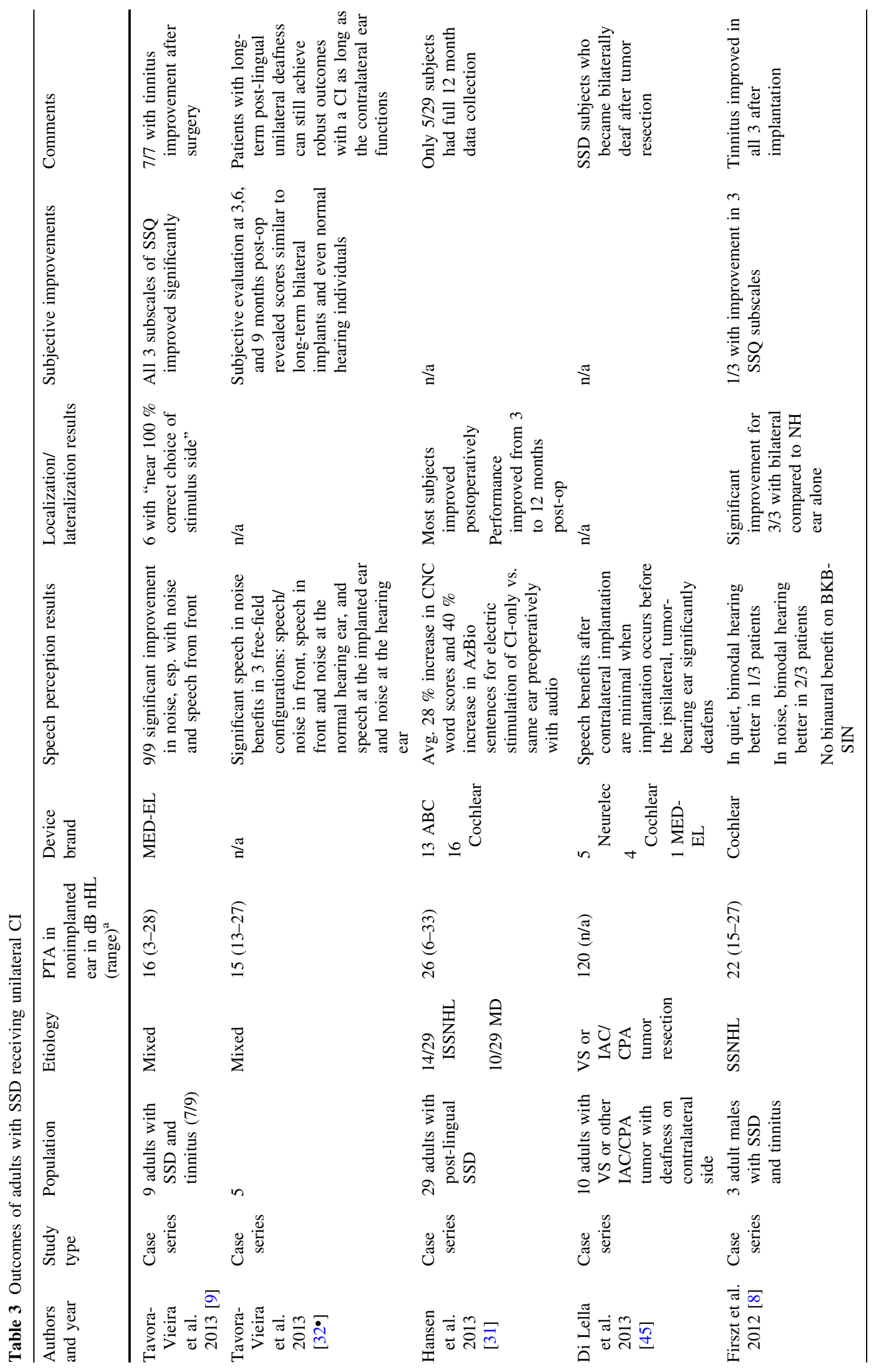




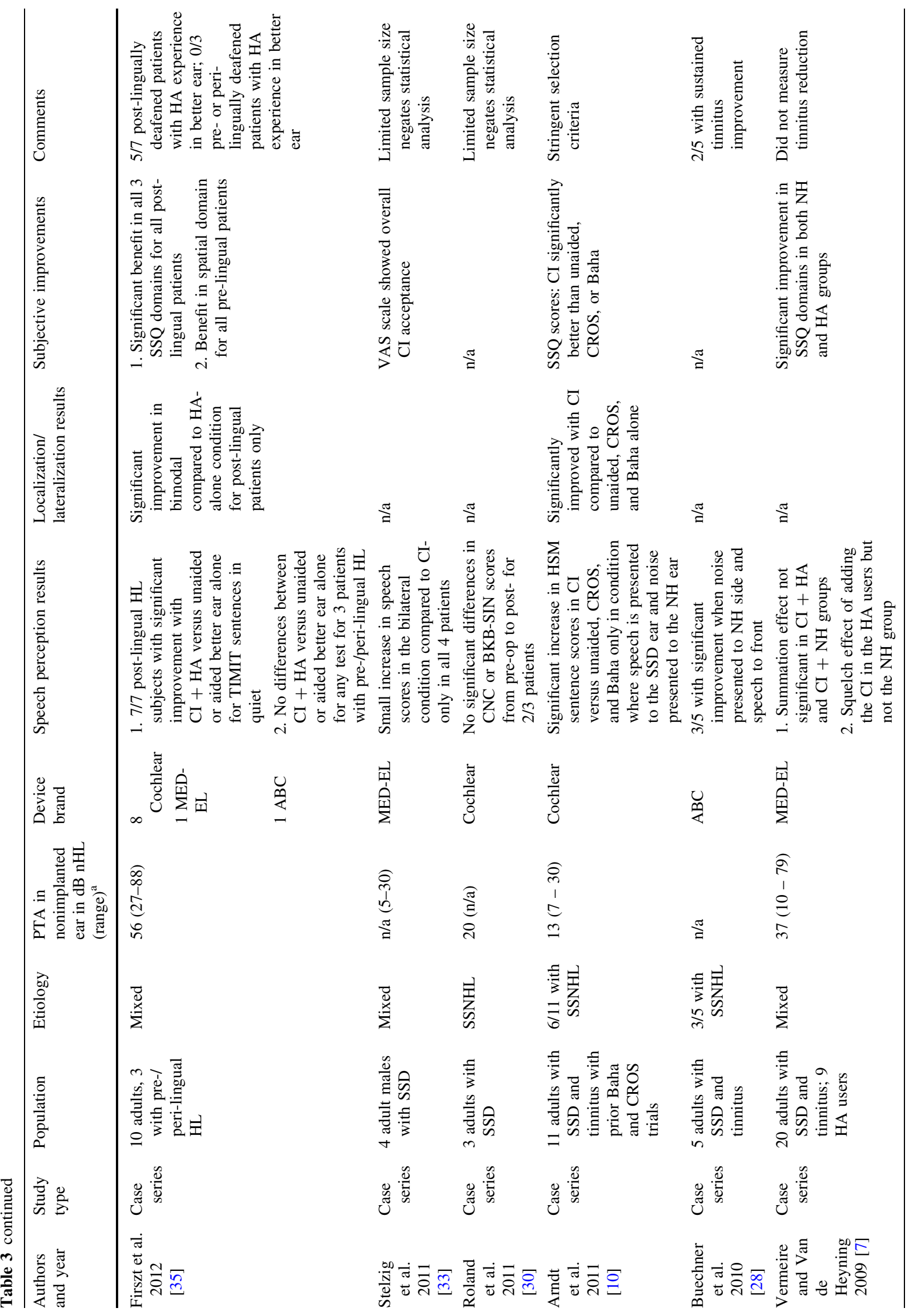




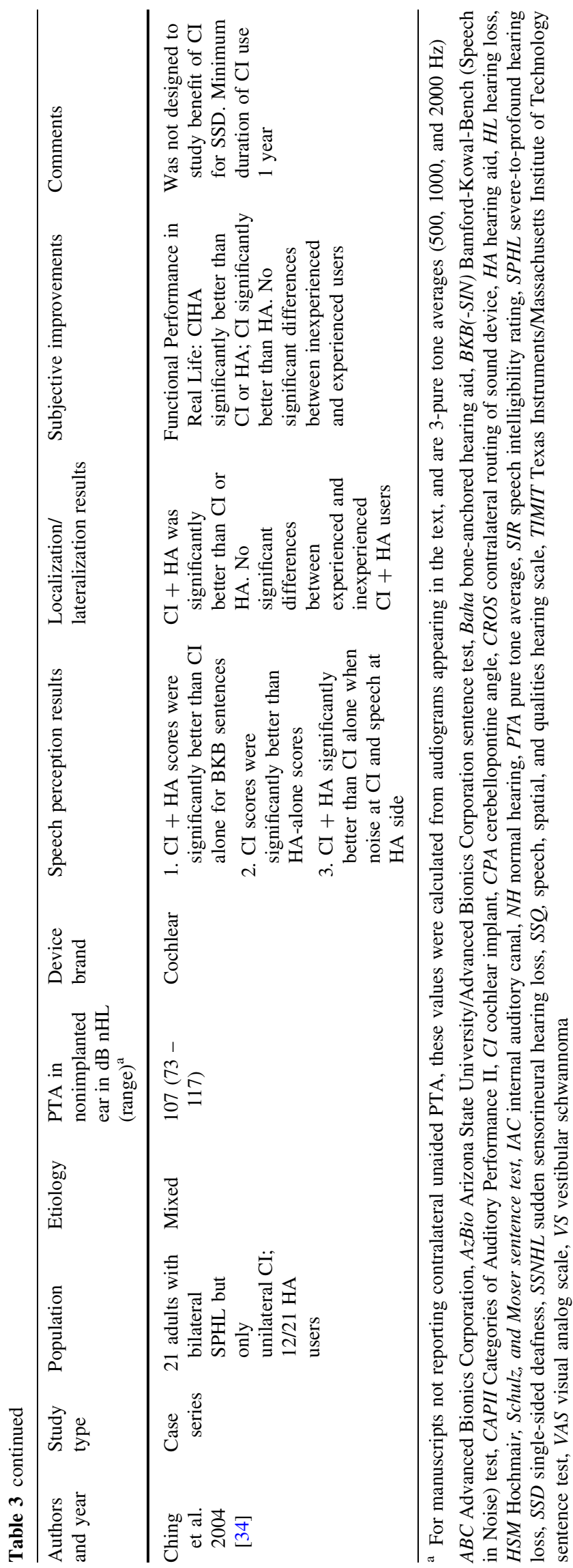

When speech and noise are presented in varying spatial configurations, the true benefit of $\mathrm{CI}$ in SSD becomes apparent. Tavora-Vieira et al. report better BKB-SIN speech comprehension with CI-on versus CI-off, especially when speech and noise are presented directly in front of the listener [9]. In the CI-on state, Tavora-Vieira et al. also report improvements in speech comprehension in three free-field conditions: speech and noise in front, speech in front and noise at the $\mathrm{NH}$ ear, and speech at the CI ear and noise in the NH ear [32•]. Similarly, other groups reported that implantation afforded binaural benefits when noise was presented to the $\mathrm{NH}$ ear and speech was presented either to the SSD ear [10] or to the front [28]. Stelzig et al. assessed German Freiburg monosyllable tests, dichotic listening tests, Hochmair-Schulz-Moser (HSM) sentence tests, and Oldenburg Sentence Tests (OLSA) in noise with 4 subjects and demonstrated overall gains in the binaural hearing condition compared to unilateral CI-on condition and CI-off conditions [33].

While most studies report significant gains with speech comprehension in noisy environments, controversy still exists around the utility of CI in SSD for all patients [32•]. Roland et al. report that only 1 of 3 of their study subjects receiving a CI for SSD showed significant improvements in the free-field BKN-SIN comprehension test when comparing preoperative scores to postoperative CI-activated scores [30]. Overall, however, the argument for the advantage of unilateral $\mathrm{CI}$ with respect to speech in noise is thoroughly convincing. A recent and robust meta-analysis of speech comprehension in various noisy environments by Vlastarakos et al. reports CI insertion in the SSD ear leads to better speech perception when speech is presented directly to the SSD ear or from the front, while concurrent noise is presented to the front or at the normal hearing ear (Fig. 1) $[11 \bullet \bullet$.

In patients with asymmetric hearing loss, the addition of a contralateral hearing aid is beneficial as long as a postlingual onset of hearing loss is present. Ching et al. report that the $\mathrm{CI}+$ contralateral HA condition affords better BKB sentence scores than either the CI-only or HA-only cases when speech was presented to the CI side and noise to the HA side [34]. Vermeire and Van de Heyning demonstrate that contralateral hearing aids significantly aid speech in noise due to improvements attributed mainly to the squelch effect [7]. However, Firszt et al. demonstrate speech gains in implantation for pre-lingually deaf subjects are less robust than post-lingually implanted subjects [35].

In children the speech benefits after implantation for SSD are profound, although the number of studies is limited (Table 2). Plontke et al. present a case where a child with a lateral skull base fracture and imminent fibrosis was implanted [36]. Improvements in speech discrimination in both quiet and noise, localization, and patient satisfaction 


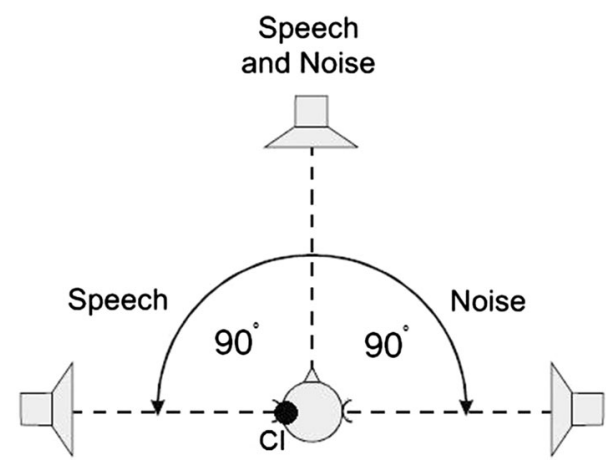

Fig. 1 Benefits of Binaural hearing after CI implantation are most beneficial when resolving speech in noise. Specifically, maximal benefit compared to the pre-implanted condition occurs when speech is presented to the implanted ear and at angles up to the front of the listener, while concurrent noise is presented at angles between the front and the normal hearing ear. $C I$ implanted ear, contralateral ear is the normal hearing ear. Summarized from content in $\left[11^{\bullet \bullet}\right]$

were reported. To assess CI efficacy in various pediatric age groups, Hassepass et al. performed HSM sentence tests and (OLSA) in noise in 3 children/adolescents aged 4, 10, and 11 [2••]. Differences in speech resolution, localization, and subjective assessments demonstrate significant improvements in the post-implant $\mathrm{CI}$-aided condition relative to the unaided preoperative condition. In children with asymmetric hearing loss, Cadieux et al. report significant $\mathrm{CNC}$ and BKBSIN speech improvements when children listened bimodally versus CI-only or HA-only conditions [37]. Concordant with Cadieux, Tzifa and Hanvey report improvements in CAPII (7/7) and SIR (6/8) scores in children with asymmetric hearing loss utilizing an implant [38].

\section{Sound Localization Benefits after Unilateral Cochlear Implantation}

Because binaural hearing is necessary for horizontal sound localization, unilateral CI drastically improves a SSD subject's ability to identify sound sources. Outcomes of localization in adults are compared in Table 3. In patients with adequate localization ability in the HA-only condition, binaural benefit from CI was limited. However, in patients where localization was poor pre-implant, localization increased significantly. At a basic level, patients implanted for SSD are able to recognize on which side (CI ear or $\mathrm{NH}$ ear) sound was being presented to [9]. Firszt et al. found significant improvements in localization with CI compared to HA-only localization [35]. Additionally, patients with CIs performed significantly better with their CI when localizing than in unaided, CROS, and Baha conditions [10]. In a case series of 3 patients, Firszt et al. demonstrated improvements in localization in $10^{\circ}$ increments when sound was presented to the $\mathrm{CI}$ ear, $\mathrm{NH}$ ear, and bilaterally [8].

A thorough meta-analysis of implantation in SSD patients found subjective improvement in localization, and improvements in localization when sound sources were presented to the CI ear at angles approaching $90^{\circ}$ to the front of the patient, even when noise was presented to the contralateral quartile of the normal hearing ear [11••]. Hansen et al. found that the greatest improvements in localization were during the 3-6 months postoperative period, inferring an experience dependence [31]. However, the study was limited because only 5 of the 29 patients had full localization assessments through the entire 12-month trial as it was designed. In patients with asymmetric hearing loss, Ching et al. showed that adding a contralateral hearing aid improved localization compared to CI-alone or HA-alone groups [34]. It is interesting to note that localization ability is maximized when ears are matched by bilateral acoustic (HA) or bilateral electric (CI) input, compared to unilateral CI and contralateral HA [39].

Localization benefits are slightly better in adults than children [40], though the number of studies quantifying localization ability in children is limited (Table 1). In a study with 3 children aged 4,10 , and 11, localization of the older two children was assessed by presenting OLSA sentences in 7 equidistant speakers placed at $30^{\circ}$ increments [2••]. Localization deviation from the source decreased in both of the two subjects who were tested. In noisy conditions, Plontke et al. reported improvements in angle detection error when the noise was presented frontally or to the CI side [36]. Finally, Cadieux et al. demonstrate that for $3 / 5$ children implanted with asymmetric hearing loss, adding a contralateral hearing aid significantly increased hearing ability compared to the CI-alone or HAalone conditions [37].

\section{Unilateral Cochlear Implant Candidacy}

\section{Children with Single-Sided Deafness}

Although adult outcome studies by far outnumber pediatric reports, the argument to implant in children appears robust. As such, children with SSD should be implanted-ideally as soon as possible because the best outcomes in SSD children have been associated with early implantation [38]. Many children with SSD demonstrate delays in speech and language comprehension [41, 42] as well as an increased likelihood of academic difficulty [2••, 43]. As unilateral hearing requires an increased effort to comprehend speech in noisy situations, it is not unsurprising that a 2010 study of prospectively followed children with SSD demonstrated they had attention fatigue, behavioral problems, and academic 
weakness in $25 \%$ compared with bilaterally-hearing peers [44]. Children with post-lingually acquired SSD who were implanted with a CI performed objectively better with speech in noise and localization, and subjectively better in their perceived hearing ability or patient satisfaction [2••, 36]. Early detection of SSD in children seems a key factor in subsequent speech perception outcomes, as intervention at a young age allows for greater neural plasticity to take advantage of input from the implanted ear. Further, in cases where a second CI is indicated in the contralateral ear, as in the case of progressive hearing loss, cat models suggest that only the total duration of bilateral deafness (i.e. time to first implant) is associated with auditory outcomes [4].

In children for whom the unilaterally deafened ear does not respond to amplification, cochlear implants clearly show substantial benefits in speech, academic performance, and quality of life. Similar to CI in bilaterally profoundly hearing impaired children, the decision to undergo implantation hinges on parental investment in, and institutional availability of, postoperative auditory rehabilitation with specialized pediatric personnel so that children may maximize the auditory gain from their devices. These factors should be weighed against the speech and behavioral gains associated with implanting a child with SSD.

\section{Adults with Single-Sided Deafness}

While studies of children consistently show psychosocial and behavioral improvements after implantation, the claim that implanting every adult will conclusively bring benefits is not as definitive. For example, the addition of an implant and restoration of binaural input seems less efficacious in prelingually deafened adults [35]. The extent to which unilateral deafness affects an individual's activities of daily living, and the capacity that binaural benefits will significantly affect his or her quality of life, must be weighed on a personalized level.

Interestingly, auditory neural pathways are preserved bilaterally as long as the only hearing ear has substantial ability to stimulate unilateral auditory pathways [32•]. In a unique meta-analysis of patients with SSD and slowly growing vestibular schwannomas in the contralateral (normal hearing) ear, only duration of total bilateral deafness was associated with hearing outcomes [45]. One recent study even showed drastic speech improvements in noise in patients with unilateral deafness as long as 40 years before single-sided implantation [9, 32 - ]. As such, unlike bilateral deafness, duration of unilateral deafness has not been definitively associated with implant outcomes - so there is less of a time sensitivity to make the decision to implant if a patient is unsure. However, the decision to implant may be expedited by concurrent medical conditions which require surgery in the neighboring anatomical areas, such as implantation during an indicated labyrinthectomy in patients with recalcitrant Ménière's Disease in the treatment of vertigo [31].

\section{Single-Sided Deafness with Ipsilateral Tinnitus}

The first accounts of CI implantation serendipitously reducing tinnitus in SNHL patients were reported in 1976 by House et al. [46] and later characterized by Brackmann in 1981 [47], despite tinnitus not being the primary indication for implantation. Over the past decade, in patients with SSD and concurrent ipsilateral debilitating tinnitus, the main indication to implant is more of an attempt to suppress the tinnitus than to strictly replace hearing. Indications for implanting a patient with SSD for the primary goal of suppressing tinnitus were thoroughly analyzed by Punte, Meeus, and Van de Heyning, with main inclusion criteria being severe tinnitus for less than a decade and concurrent ipsilateral SNHL, and main exclusion criteria being patient's inability/unwillingness to attend regular follow-up and CI rehabilitation [48]. With recent knowledge that a $\mathrm{CI}$-induced reduction of tinnitus in the SSD ear increases speech perception in the $\mathrm{NH}$ ear, indications for treating SSD are expanding [49]. A thorough meta-analysis of recent literature relating to $\mathrm{CI}$ in treating unilateral SSD patients with tinnitus was authored by Arts et al. [50•], who showed significant decreases in tinnitus after implantation assessed by Visual Analog Scale (VAS) at 1-, 3-, 6-, and 24-month post-op compared to the tinnitus VAS levels in the pre-implant condition.

While traditional attempts to preserve the integrity of the cochlear apex by using short CIs may be beneficial in the patient with preserved low-frequency thresholds, using a short electrode cannot be recommended if the patient has ipsilateral tinnitus because full-length cochlear stimulation may be necessary to suppress tinnitus [51]. Although the suspected mechanisms of tinnitus reduction after implantation include habituation and cochlear reorganization [52], research is warranted to develop CI stimulus algorithms specifically aimed at suppressing tinnitus. Studies describe modified CI stimulus paradigms with both high frequency [53] and low-frequency [54] currents optimally suppressing tinnitus in case reports, and variable outcomes of tinnitus suppression with standard stimulus patterns [28].

\section{Improving Postoperative Outcomes in Single-Sided} Deafness Patients

Major contributing factors to post-implant outcomes are the attempts to preserve hearing during implantation, therapy during the postoperative auditory neuroplastic window, and the use of additional acoustic amplifiers. Preserving hearing via surgical techniques can reduce trauma to the cochlea and ultimately aid in speech and localization benefits. Postoperatively, the auditory cortex demonstrates the greatest extent 
of reorganization during the first 6 months post-activation, so it is crucial to keep implanted subjects' motivation high during this critical period of speech therapy [55]. In addition to training, combined electro-acoustic stimulation with an acoustic amplifier may be beneficial in certain populations. In cases where subjects have some degree of preserved lowfrequency hearing, an acoustic amplifier in the ipsilateral ear may assist in the detection of low-frequency tones postimplant [56]. When asymmetric bilateral hearing loss exists, combining an amplifier in the contralateral ear is associated with increased hearing abilities with respect to speech in noise in both children [37, 38] and adults [2••].

\section{Conclusions}

While hearing aids and similar amplifiers are reasonable strategies for initially attempting to correct hearing loss with minimal risk to the patient, $\mathrm{CI}$ is the definitive treatment for replacing a nonfunctioning cochlea and providing binaural hearing benefits. Squelch and summation aid in increasing the signal-to-noise ratio of incoming sounds and interaural comparisons assist in localization after the head shadow effect is eliminated. Because the treatment progression from hearing aids to CI is a large decision for both patients and providers, it is important to evaluate the most recent information regarding who will benefit most. The anticipated benefit of implantation, namely speech in noise, localization, and a decreased effort to hear, should be weighed on an individualized level against the potential risks. For children, the argument to implant is compelling. For adults, the duration of unilateral deafness may not be as strong a contributor to outcomes as duration of bilateral deafness, so the decision to implant may not require quick judgment.

\section{Compliance with Ethics Guidelines}

Conflict of Interest Christopher K. Giardina, Eric J. Formeister, and Oliver F. Adunka have declare no conflict of interest.

Human and Animal Rights and Informed Consent This article does not contain any studies with human or animal subjects performed by the authors.

\section{References}

Papers of particular interest, published recently, have been highlighted as:

- Of importance

-• Of major importance

1. Ross DS, et al. Highly variable population-based prevalence rates of unilateral hearing loss after the application of common case definitions. Ear Hear. 2010;31(1):126-33.
2. • Hassepass, F., et al. Unilateral deafness in children: audiologic and subjective assessment of hearing ability after cochlear implantation. Otology \& Neurotology, 2013. 34(1):53-60. Case series regarding $C I$ in $S S D$ children.

3. Mehl AL, Thomson V. The colorado newborn hearing screening project, 1992-1999: on the threshold of effective population-based universal newborn hearing screening. Pediatrics. 2002;109(1):e7.

4. Kral A, Hubka P, Heid S, Tillein J. Single-sided deafness leads to unilateral aural preference within an early sensitive period. Brain. 2013;136(Pt 1):180-93.

5. Northern JL, Downs MP. Hearing in children. Philadelphia: Lippincott Williams \& Wilkins; 2002.

6. - Kamal, S.M., A.D. Robinson, and R.C. Diaz. Cochlear implantation in single-sided deafness for enhancement of sound localization and speech perception. Current Opinion in Otolaryngology \& Head and Neck Surgery. 2012; 20(5):393-397. Thorough overview of binaural mechanisms and benefits of unilateral CI in SSD.

7. Vermeire K, de Heyning PV. Binaural hearing after cochlear implantation in subjects with unilateral sensorineural deafness and tinnitus. Audiol Neuro-Otol. 2009;14(3):163-71.

8. Firszt JB, et al. Auditory abilities after cochlear implantation in adults with unilateral deafness: a pilot study. Otol Neurotol. 2012;33(8):1339-46.

9. Tavora-Vieira D, et al. Cochlear implantation for unilateral deafness with and without tinnitus: a case series. Laryngoscope. 2013;123(5):1251-5.

10. Arndt $\mathrm{S}$, et al. Comparison of pseudobinaural hearing to real binaural hearing rehabilitation after cochlear implantation in patients with unilateral deafness and tinnitus. Otol Neurotol. 2011;32(1):39-47.

11. • Vlastarakos, P.V., et al., Cochlear implantation for single-sided deafness: the outcomes. An evidence-based approach. Eur Arch Otorhinolaryngol. 2013. Important review for systematically evaluating outcomes of CI for SSD in various research groups.

12. Bishop CE, Eby TL. The current status of audiologic rehabilitation for profound unilateral sensorineural hearing loss. Laryngoscope. 2010;120(3):552-6.

13. Hill SL 3rd, et al. Assessment of patient satisfaction with various configurations of digital CROS and BiCROS hearing aids. Ear Nose Throat J. 2006;85(7):427-30.

14. Lin LM, et al. Amplification in the rehabilitation of unilateral deafness: Speech in noise and directional hearing effects with bone-anchored hearing and contralateral routing of signal amplification. Otol Neurotol. 2006;27(2):172-82.

15. Valente M, et al. Wireless CROS versus transcranial CROS for unilateral hearing loss. Am J Audiol. 1995;4:52-9.

16. Hol MKS, et al. Pilot study on the effectiveness of the conventional CROS, the transcranial CROS and the BAHA transcranial CROS in adults with unilateral inner ear deafness. Eur Arch Otorhinolaryngol. 2010;267(6):889-96.

17. Nicolas S, et al. Long-term benefit and sound localization in patients with single-sided deafness rehabilitated with an osseointegrated bone-conduction device. Otol Neurotol. 2013;34(1):111-4.

18. Hol MKS, et al. Bone-anchored hearing aids in patients with acquired and congenital unilateral inner ear deafness (Baha CROS): clinical evaluation of 56 cases. Ann Otol Rhinol Laryngol. 2010;119(7):447-54.

19. Yuen HW, et al. Management of single-sided deafness with the bone-anchored hearing aid. Otolaryngol-Head and Neck Surg. 2009;141(1):16-23.

20. Niparko JK, Cox KM, Lustig LR. Comparison of the bone anchored hearing aid implantable hearing device with contralateral routing of offside signal amplification in the rehabilitation of unilateral deafness. Otol Neurotol. 2003;24(1):73-8.

21. Wazen JJ, et al. Transcranial contralateral cochlear stimulation in unilateral deafness. Otolaryngol-Head and Neck Surg. 2003;129(3):248-54. 
22. Baguley DM, et al. The evidence base for the application of contralateral bone anchored hearing aids in acquired unilateral sensorineural hearing loss in adults. Clin Otolaryngol. 2006; 31(1):6-14.

23. Djourno A, Eyriès C. Prothese auditive par excitation electrique a distance du nerf sensoriel a laide dun bobinage inclus a demeure. Presse médicale. 1957;65(63):1417.

24. Simmons FB, et al. Auditory nerve: electrical stimulation in man. Science. 1965;148(3666):104-6.

25. House WF, Urban J. Long term results of electrode implantation and electronic stimulation of the cochlea in man. Ann Otol Rhinol Laryngol. 1973;82(4):504.

26. de Heyning PV, et al. Incapacitating unilateral tinnitus in singlesided deafness treated by cochlear implantation. Ann Otol Rhinol Laryngol. 2008;117(9):645-52.

27. Arts RAGJ, et al. Review: cochlear implants as a treatment of tinnitus in single-sided deafness. Curr Opin Otolaryngol Head and Neck Surg. 2012;20(5):398-403.

28. Buechner A, et al. Cochlear implantation in unilateral deaf subjects associated with ipsilateral tinnitus. Otol Neurotol. 2010;31(9):1381-5.

29. Jacob R, Stelzig Y. The koblenz experience in treating singlesided deafness with cochlear implants. Audiol Neurotol. 2011;16(Suppl 1):6-8.

30. Roland JT, Shapiro WH, Waltzman S. Cochlear implantation as a treatment option for single-sided deafness: speech perception benefit. Audiol Neurootol. 2011;16(suppl 1):8-9.

31. Hansen MR, Gantz BJ, Dunn C. Outcomes after cochlear implantation for patients with single-sided deafness, including those with recalcitrant meniere's disease. Otol Neurotol. 2013;34(9):1681-7.

32. - Tavora-Vieira, D., et al., Successful outcomes of cochlear implantation in long-term unilateral deafness: brain plasticity? Neuroreport. 2013. 24(13):724-729. Important study challenging the preception that duration of SSD is associated with CI outcomes.

33. Stelzig Y, Jacob R, Mueller J. Preliminary speech recognition results after cochlear implantation in patients with unilateral hearing loss: a case series. J Med Case Rep. 2011;5:343.

34. Ching TY, Incerti P, Hill M. Binaural benefits for adults who use hearing aids and cochlear implants in opposite ears. Ear Hear. 2004;25(1):9-21.

35. Firszt JB, et al. Cochlear implantation in adults with asymmetric hearing loss. Ear Hear. 2012;33(4):521.

36. Plontke SK, et al. Cochlear implantation in a child with posttraumatic single-sided deafness. Eur Arch Otorhinolaryngol. 2013;270(5):1757-61.

37. Cadieux JH, Firszt JB, Reeder RM. Cochlear implantation in nontraditional candidates: preliminary results in adolescents with asymmetric hearing loss. Otol Neurotol. 2013;34(3):408-15.

38. Tzifa K, Hanvey K. Cochlear implantation in asymmetrical hearing loss for children: our experience. Cochlear Implants Int. 2013;14(S4):56-61.
39. Gifford RH, et al. Localization and interaural time difference (ITD) thresholds for cochlear implant recipients with preserved acoustic hearing in the implanted ear. Hear Res. 2014;312:28-37.

40. Jacob R, et al. Audiological results with cochlear implants for single-sided deafness. HNO. 2011;59(5):453-60.

41. Wiley S, Arjmand E, Dixon M. Findings from multidisciplinary evaluation of children with permanent hearing loss. Int J Pediatr Otorhinolaryngol. 2011;75(8):1040-4.

42. McKay S, Gravel JS, Tharpe AM. Amplification considerations for children with minimal or mild bilateral hearing loss and unilateral hearing loss. Trends in Amplif. 2008;12(1):43-54.

43. Morita S, Suzuki M, Iizuka K. Non-organic hearing loss in childhood. Int J Pediatr Otorhinolaryngol. 2010;74(5):441-6.

44. Lieu JE, Tye-Murray N, Fu Q. Longitudinal study of children with unilateral hearing loss. The Laryngoscope. 2012;122(9): 2088-95.

45. Di Lella F, et al. Vestibular Schwannoma in the Only Hearing Ear: Role of Cochlear Implants. Ann Otol Rhinol Laryngol. 2013;122(2):91-9.

46. House WF. Cochlear implants. Ann Otol Rhinol Laryngol. 1976;85(3Pt2):1.

47. Brackmann D. Reduction of tinnitus in cochlear-implant patients. J laryngol Otol. 1981;4:163.

48. Punte AK, Meeus O, Van de Heyning P. Cochlear implants and tinnitus. Textbook of tinnitus. Heidelberg: Springer; 2011. p. 619-24.

49. Mertens G, et al. Tinnitus in a single-sided deaf ear reduces speech reception in the nontinnitus ear. Otol Neurotol. 2013;34(4):662-6.

50. - Arts, R.A., et al., Review: cochlear implants as a treatment of tinnitus in single-sided deafness. Current opinion in otolaryngology \& head and neck surgery, 2012. 20(5):398-403. Important review of outcomes after CI insertion in patients with SSD and concurrent ipsilateral tinnitus.

51. Kleine Punte A, De Ridder D, Van de Heyning P. On the necessity of full length electrical cochlear stimulation to suppress severe tinnitus in single-sided deafness. Hear Res. 2013;295: 24-9.

52. Ramos Á, et al. Cochlear implant in patients with sudden unilateral sensorineural hearing loss and associated tinnitus. Acta Otorrinolaringologica (English Edition). 2012;63(1):15-20.

53. Rubinstein JT, et al. Electrical suppression of tinnitus with highrate pulse trains. Otol Neurotol. 2003;24(3):478-85.

54. Zeng F-G, et al. Tinnitus suppression by low-rate electric stimulation and its electrophysiological mechanisms. Hear Res. 2011;277(1):61-6.

55. Pantev C, et al. Dynamics of auditory plasticity after cochlear implantation: a longitudinal study. Cereb Cortex. 2006;16(1): 31-6.

56. von Ilberg CA, et al. Electric-acoustic stimulation of the auditory system: a review of the first decade. Audiol Neurotol. 2011;16(Suppl. 2):1-30. 